\title{
Space charge and charge trapping characteristics of cross-linked polyethylene subjected to ac electric stresses
}

\author{
Y L Chong ${ }^{1}$, G Chen ${ }^{1}$, H Miyake ${ }^{2}$, K Matsui ${ }^{2}$, Y Tanaka ${ }^{2}$ and \\ T Takada ${ }^{2}$ \\ ${ }^{1}$ School of Electronics and Computer Science, University of Southampton, Southampton, \\ SO17 1BJ, UK \\ ${ }^{2}$ Electronics Measurement Laboratory, Musashi Institute of Technology, 1-28-1 \\ Tamazutsumi, Setagaya-ku, Tokyo 158-8557, Japan
}

Received 6 October 2005, in final form 13 February 2006

Published 30 March 2006

Online at stacks.iop.org/JPhysD/39/1658

\begin{abstract}
This paper reports on the result of space charge evolution in cross-linked polyethylene (XLPE) planar samples approximately $220 \mu \mathrm{m}$ thick. The space charge measurement technique used in this study is the pulsed electroacoustic method.

There are two phases to this experiment. In the first phase, the samples were subjected to dc $30 \mathrm{kV}_{\mathrm{dc}} \mathrm{mm}^{-1}$ and ac (sinusoidal) electric stress levels of $30 \mathrm{kV}_{\mathrm{pk}} \mathrm{mm}^{-1}$ at frequencies of 1,10 and $50 \mathrm{~Hz}$ ac. In addition, ac space charge under $30 \mathrm{kV}_{\mathrm{rms}} \mathrm{mm}^{-1}$ and $60 \mathrm{kV}_{\mathrm{pk}} \mathrm{mm}^{-1}$ electric stress at $50 \mathrm{~Hz}$ was also investigated. The volts-off results showed that the amount of charge trapped in XLPE sample under dc electric stress is significantly bigger than samples under ac stress even when the applied ac stresses are substantially higher.

The second phase of the experiment involves studying the dc space charge evolution in samples that were tested under ac stress during the first phase of the experiment. Ac ageing causes positive charge to become more dominant over negative charge. It was also discovered that ac ageing creates deeper traps, particularly for negative charge.

This paper also gives a brief overview of the data processing methods used to analyse space charge under ac electric stress.
\end{abstract}

(Some figures in this article are in colour only in the electronic version)

\section{Introduction}

Over the past two decades, space charges within dielectrics under dc electric stress (herein known as dc space charge) have received much interest and numerous literature regarding their evolution and activities in different types of materials with different chemical additives were published [1-7].

On the other hand, investigation in space charges under $50 \mathrm{~Hz}$ ac condition remains a relatively unexplored field. Indeed, it is only in the last five years or so that results, albeit limited, of space charge in polymeric insulation subjected to ac stress (herein known as ac space charge) have been reported [8-12].

Various measurement techniques, formerly only used for dc space charge, such as the thermal step method [9], the pulsed electroacoustic (PEA) method [10-12] and the laser induced pressure pulse (LIPP) method [8], were employed for ac space charge measurements.

Earlier results using the PEA technique [10] revealed that the critical frequency on which space charge accumulates is $<0.02 \mathrm{~Hz}$. However, findings in $[9,13]$ have shown clear evidence of space charge accumulation in polymeric 
Characteristics of cross-linked polyethylene subjected to ac electric stresses

insulation when stressed at power frequency $(50 \mathrm{~Hz})$. It should be pointed out that the poling times were considerably longer in the literature whereby space charge accumulations were observed. It was also suggested in [13] that different electrode/insulation interface could be another factor contributing to the inconsistency in the literature. Indeed, it was demonstrated that ac space charge of a very different nature (in fact completely opposite) was observed with different electrode/insulation interface configurations.

This paper reports on the result of space charge evolution in cross-linked polyethylene (XLPE) planar samples approximately $220 \mu \mathrm{m}$ thick. The space charge measurement technique used in this study is the PEA method.

There are two phases to this experiment. First, the samples were subjected to dc $30 \mathrm{kV}_{\mathrm{dc}} \mathrm{mm}^{-1}$ and ac (sinusoidal) electric stress level of $30 \mathrm{kV}_{\mathrm{pk}} \mathrm{mm}^{-1}$ at frequencies of 1,10 and $50 \mathrm{~Hz}$ ac for $24 \mathrm{~h}$. In addition, ac space charge under $30 \mathrm{kV}_{\mathrm{rms}} \mathrm{mm}^{-1}$ and $60 \mathrm{kV}_{\mathrm{pk}} \mathrm{mm}^{-1}$ electric stress at $50 \mathrm{~Hz}$ was also investigated.

Space charge mechanism in dielectric under ac electric stress is often difficult to understand as numerous factors such as the varying amplitude of sinusoidal ac voltage, voltage reversal, charging trapping/cancellation, charge injection/extraction, material degradation, etc need to be considered. Hence, the second phase of the experiment involves studying the dc space charge evolution in samples that were pre-aged under ac stress.

\section{Experimental details}

\subsection{Automated PEA system}

PEA is by far the most widely used technique in the field of space charge measurements. This technique utilizes interaction between high voltage pulses and charge layers accumulated in the insulating material to produce acoustic pressure waves which traverse across the material. Detailed reviews on the principle of PEA can be found in the literature $[14,15]$. To summarize, acoustic pressure waves are produced when an electrical pulse, applied externally, interacts with charge layers at the electrodes and/or in the material. The acoustic waves, which are proportional to the charge layers, are converted into an electrical signal by a piezo-electric transducer, amplified and captured with a digitial oscilloscope.

Due to the frequency behaviour of the piezo-electric transducer and amplifer, distortion of the output signal occurs as shown in figure 1 .

The peak between the cathode and anode, as seen in figure 1 , is not due to space charge but rather the frequency response of the system. Nonetheless, a quantitative description of charge across the sample can be obtained through a deconvolution technique and calibration [16].

Furthermore, as the acoustic wave propagates through a material, the absorption and dissipation of the acoustic energy into heat and other forms of energy cause attenuation of the signal. However, the inhomogeneities and frequency dependence of the material constants such as Young's modulus may result in dispersion of the acoustic signal. A signal recovery algorithm involving a Fourier transform has been successfully empolyed to compensate such effects in thick

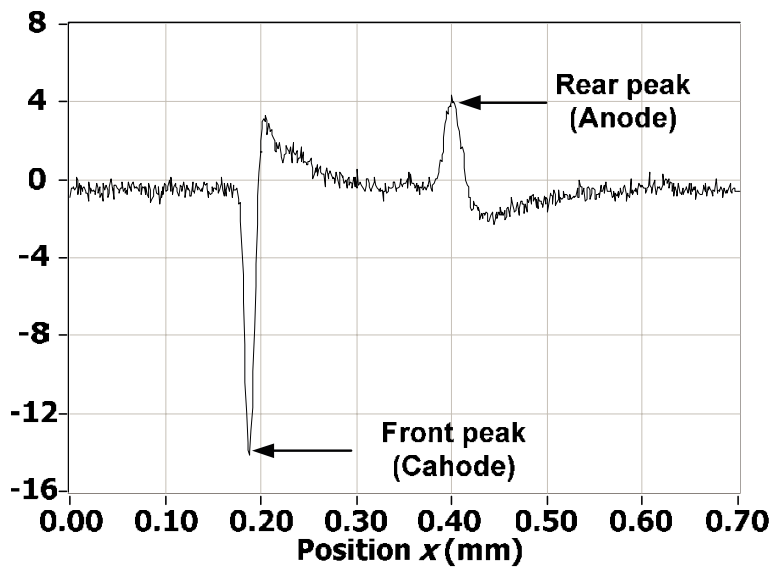

Figure 1. A typical PEA output signal.

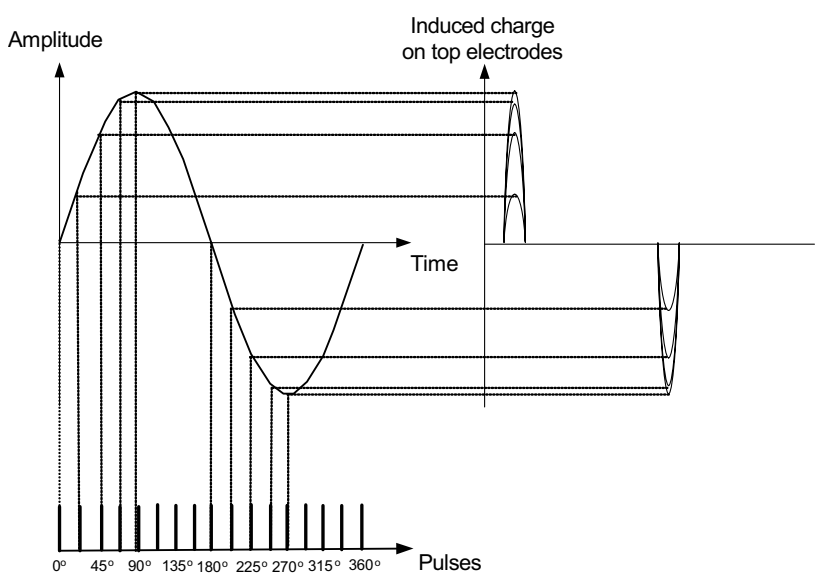

Figure 2. Point-on-wave measurement.

samples [17]. However, in thin samples such as the ones used in this study, such effects are usually considered to be negligible.

In the case of dc space charge, the applied voltage is not time dependent. However, for ac space charge, the applied voltage varies with time. It is therefore important to correlate the space charge measured with the applied ac voltage. This is best achieved with the 'point-on-wave' method. The principle of the 'point-on-wave' method is shown in figure 2. The detailed description of the PEA method under ac voltage has been described elsewhere $[18,19]$.

As illustrated in figure 2, measurement on a different phase of the ac waveform will yield an output of different magnitude and polarity for the induced charge on the electrode. For clarity, traces of the induced charge on the top electrode are shifted to the right when polarity is changed.

Figure 3 shows the schematic diagram of the fully automated PEA system.

In conventional PEA system, the time required to obtain a space charge measurement under dc electric stress is typically about $30 \mathrm{~s}$. However, this improved PEA system is able to obtain a measurement in less than $1 \mathrm{~s} \mathrm{[6].} \mathrm{This} \mathrm{is} \mathrm{especially}$ useful when space charge dynamics at short time intervals are to be studied.

With reference to figure 3 , the computer is used to generate $\mathrm{dc}$ or ac waveforms of various amplitudes and frequencies. 


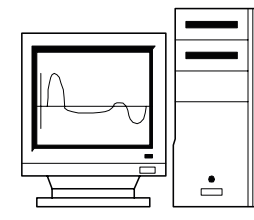

Computer

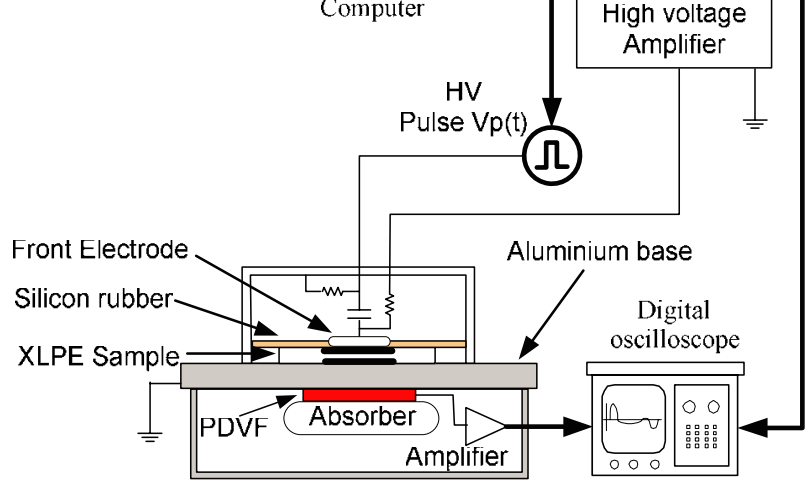

Figure 3. Schematic diagram of the pulsed electroacoustic technique.

Such a waveform is then fed into a high voltage amplifier and finally across the sample. An electric pulse of $10 \mathrm{~ns}$ duration and $2 \mathrm{kV}$ amplitude is applied to the sample to generate the acoustic signal. Since the high voltage pulses required to generate the pressure waves are also controlled by the computer, it is possible to apply the high voltage pulses on the desired points of the ac waveform with precision to achieve the 'point-on-wave' measurement approach.

This is a different approach from the one used in the LIPP system as reported in [8]. In the LIPP system, the voltage across the sample was monitored by a capacitor divider while the timing of the measurements was controlled with an electronic control board.

\subsection{Sample details}

The test samples used in this study were planar samples consisting of XLPE insulation with semicon electrodes hotpressed on either sides. The insulating bulk was made from cable grade XLPE (Borealis XL4201S), while the semicon was made of the same grade of polyethylene material, but was loaded with carbon black to increase conductivity (Borealis LEO592).

Both the insulation of $\sim 400 \mu \mathrm{m}$ thick and the semicons of $\sim 200 \mu \mathrm{m}$ thick were made separately at temperatures below the activation threshold of the crosslinking agent-dicumyl peroxide (DCP).

Crosslinking was achieved by hot-pressing the electrodes into the insulating bulk at $200^{\circ} \mathrm{C}$ for about 10 min, ensuring a good interfacial contact at the same time. The resultant thicknesses of the samples were approximately $200 \mu \mathrm{m}$ thick in bulk insulation.

All samples were degassed in a vacuum oven at $90^{\circ} \mathrm{C}$ for $48 \mathrm{~h}$ to remove volatile by-products of the crosslinking agent.

\subsection{Experimental protocols}

In the first phase of the experiment, all samples were electrically aged for a duration of $24 \mathrm{~h}$. Space charge
Table 1. Experimental protocols (first phase).

\begin{tabular}{lccc}
\hline Sample & Treatment & Stress level & Frequency \\
\hline A & Degassed & $-30 \mathrm{kV}_{\mathrm{dc}} \mathrm{mm}^{-1}$ & $\mathrm{dc}$ \\
B & Degassed & $30 \mathrm{kV}_{\mathrm{pk}} \mathrm{mm}^{-1}$ & $1 \mathrm{~Hz}$ Sine \\
C & Degassed & $30 \mathrm{kV}_{\mathrm{pk}} \mathrm{mm}^{-1}$ & $10 \mathrm{~Hz}$ Sine \\
D & Degassed & $30 \mathrm{kV}_{\mathrm{pk}} \mathrm{mm}^{-1}$ & $50 \mathrm{~Hz}$ Sine \\
E & Degassed & $30 \mathrm{kV}_{\mathrm{rms}} \mathrm{mm}^{-1}$ & $50 \mathrm{~Hz}$ Sine \\
F & Degassed & $60 \mathrm{kV}_{\mathrm{pk}} \mathrm{mm}^{-1}$ & $50 \mathrm{~Hz}$ Sine \\
\hline
\end{tabular}

distributions were measured at hourly intervals during the ageing period with voltage applied. Details of the experimental protocols can be seen in table 1 .

Investigations on dc space charge dynamics were done mainly as a reference to assist the understanding of ac space charge dynamics since its behaviour is better understood.

The 'point-on-wave' approach is not necessary for a dc space charge since the applied voltage does not vary with time. In the case of ac space charge, however, 16 equally spaced measurements on a complete sinusoidal wave were taken. After $24 \mathrm{~h}$ of ageing, the short-circuit space charge distributions were measured and monitored for $2 \mathrm{~h}$.

The second phase of the experiment was carried out about 1 month after completion of the first. All samples that were aged under ac electric stress were stored at room temperature during the 1 month, allowing any charge trapped to decay.

After the storage period, a step voltage test was conducted to investigate the stress at which space charge initiates. In this experiment, voltage was increased from $0 \mathrm{kV}$, at a voltage step of $1 \mathrm{kV}$ every $30 \mathrm{~s}$ until a stress level of $30 \mathrm{kV}_{\mathrm{dc}} \mathrm{mm}^{-1}$ was reached. Space charge measurements were taken at every incremental voltage step. The final voltage, which depends on the sample thickness, was in the range from 6 to $7 \mathrm{kV}$. The front and rear peaks of the measured output, which are proportional to the stresses at the electrodes, were then plotted against the applied voltage.

Immediately after the step voltage test, the samples were aged under $30 \mathrm{kV}_{\mathrm{dc}} \mathrm{mm}^{-1}$ electric stress for $24 \mathrm{~h}$ at ambient temperature with, once again, space charge measurement taken at regular intervals. Just as in the first phase of the experiment, the short-circuit space charge distributions in the samples after ageing were monitored for $5 \mathrm{~h}$. For the sake of brevity the samples tested in the second phase of the experiement will be labelled with 'dc-'; for example dc-sample B, dc-sample C and so on.

\section{Results and discussion}

Figure 4 shows the space charge profiles of sample A.

With reference to figure $4(a)$, which shows the volt-on charge profile during $24 \mathrm{~h}$ ageing, homocharges, or charges of the same polarity as the electrodes, can be seen in the vicinity of both electrodes after just $1 \mathrm{~h}$ of the experiment. The amount of homocharges accumulation increased with ageing duration. This accumulation of homocharges caused the interfacial stresses to reduce, as evidenced by the decrease in the amplitude of the anode and cathode.

The accumulation of homocharges in sample A was also reflected in the volt-off space charge profiles as seen 


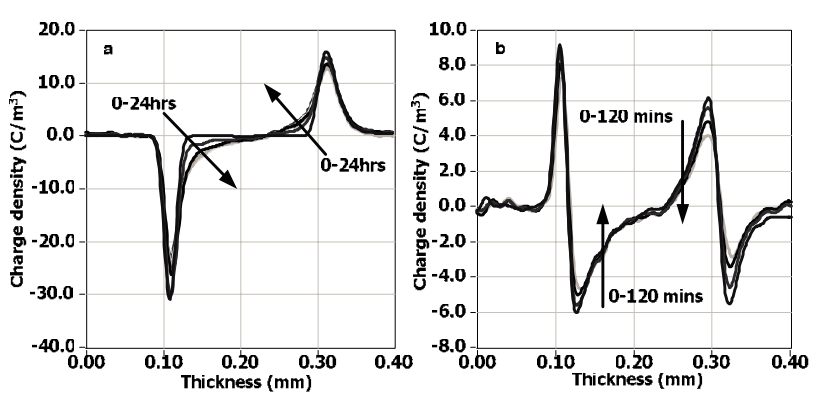

Figure 4. Charge profiles of sample A: $(a)$ volt-on $(b)$ volt-off.
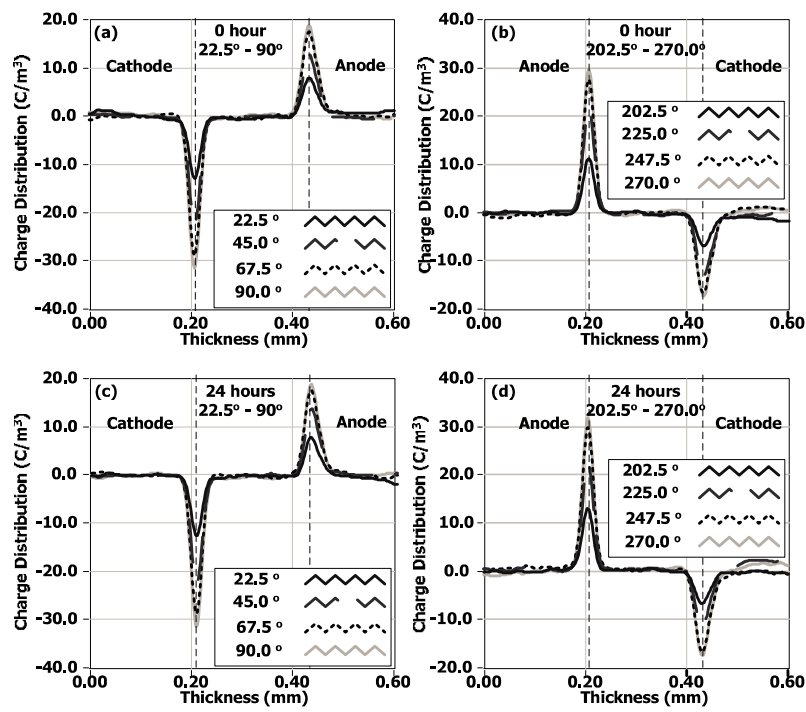

Figure 5. Charge profiles of sample B.

in figure $4(b)$. A significant decrease in space charge was observed after the applied voltage was removed for $2 \mathrm{~h}$.

The space charge of such nature is typically seen in pure LDPE or thoroughly degassed XLPE samples whereby charge injection from the electrodes is the dominant process. It was also observed that there is more negative charge than positive charge accumulated in the sample.

Figure 5 shows the space charge profiles of sample B at various phases of the ac waveform.

Figure 5 demonstrates the feasibility of the 'point-onwave' technique of the PEA system. Comparing the space charge profiles at $0 \mathrm{~h}$ with that after $24 \mathrm{~h}$ suggests negligible charge accumulation. However, a careful examination of the anodes of figures $5(b)$ and $(d)$ seems to indicate a slight increase in amplitude after $24 \mathrm{~h}$ of ageing.

As mentioned before, space charge can affect stress at the interface. As opposed to sample A, increase in stress at the anode may be caused by negative charge accumulation. Although almost no charge accumulation can be seen in figure $5(d)$, this may be due to the induced interfacial peaks masking off the substantially smaller charge accumulation.

Two methods have been proposed by Montanari et al [12] to observe space charge of such a small magnitude. The first method was to subtract the charge profile where no charge, $\rho_{\text {no-charge}}$, was accumulated from the space charge profile with

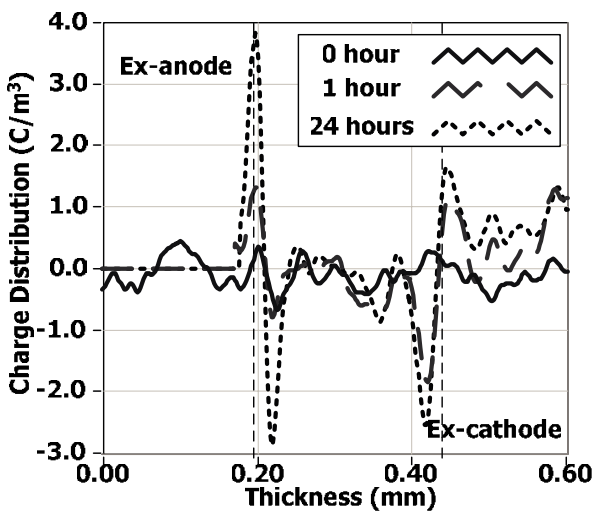

Figure 6. Charge profiles of sample B after the removal of the Laplacian contribution to induced interfacial peaks.

charge, $\rho_{\text {volt-on }}$ :

$$
\rho_{\text {accumulated }}=\rho_{\text {volt-on }}-\rho_{\text {no-charge }} \text {. }
$$

The space charge profile of $\rho_{\text {no-charge }}$ has to be obtained before the aging. First the space charge profile of the sample at a stress level that is high enough to yield good signal-to-noise ratio but small enough to avoid space charge built up in the sample was measured. The electric stress level used for this purpose is $<10 \mathrm{kV} \mathrm{mm}^{-1}$ as it has been reported that, under room temperature, threshold stress for space charge accumulation in this material is at least $10 \mathrm{kV} \mathrm{mm}^{-1}$ [7]. This space charge profile is also used for calibration purpose and will hence be known as $\rho_{\text {calibrate. }}$.

Hence $\rho_{\text {no-charge }}$ can be calculated using

$$
\rho_{\text {no-charge }}=\frac{E_{\text {applied }}}{E_{\text {calibrate }}} \times \rho_{\text {calibrate }},
$$

where $E_{\text {applied }}$ and $E_{\text {calibrate }}$ are the applied stress and calibration stress, respectively.

Figure 6 shows the space charge profiles of sample B at $270^{\circ}$ after applying the above data algorithm.

As seen in figure 6, the charge distribution is dominated by negative charge; also the amount accumulated in the sample increases with ageing duration. These results are consistent with those reported in [11].

The accumulation of negative charge at the anode led to the increase in interfacial stress there as observed in figure 5. On the other hand, the negative charge accumulated at the cathode there should cause the interfacial stress to reduce. This effect, however, is extremely subtle as the sensitivity of the system at the cathode end is substantially lower than that at the anode with the latter being close to the piezo-electric transducer.

The second method proposed was to measure the space charge profile when the Laplacian field is zero which is at the natural zero of the ac waveform, i.e. phases $0^{\circ}$ and $180^{\circ}$.

Figure 7 shows the space charge profiles of sample B at $0^{\circ}$.

The space charge profiles taken at natural zero (only $0^{\circ}$ is shown here for brevity) of the ac waveform are similar to that seen in figure 6, only smaller in magnitude. This may be attributed to very fast charge accumulated in the sample which disappeared as soon as the electric stress across the sample fell to zero. 


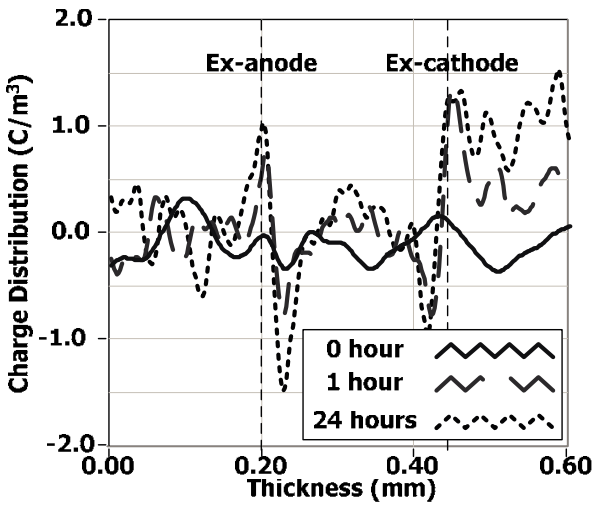

Figure 7. Charge profiles of sample B at $0^{\circ}$.

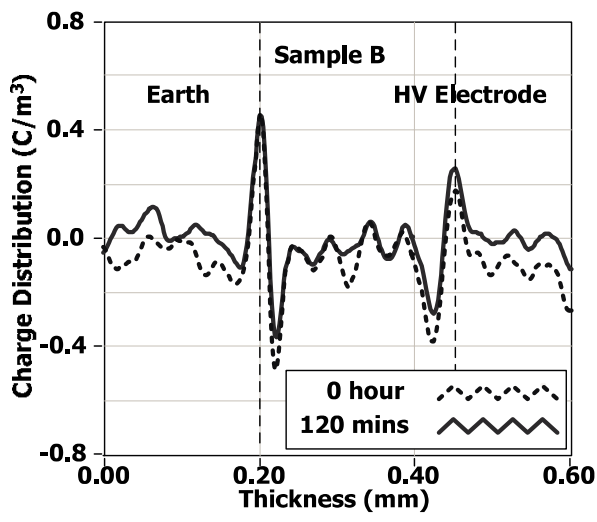

Figure 8. Volt-off charge profiles of sample B.

However, both methods are very dependent on the precision of the 'point-on-wave' technique; as such any drift in the control system may lead to erroneous results.

The 'point-on-wave' space charge profiles of samples $\mathrm{C}$ to $\mathrm{F}$ are very similar to that of sample $\mathrm{B}$, with the induced peaks masking the presence of space charge accumulation, if any at all. This suggests that the indication of space charge presence under ac conditions is extremely difficult unless there is a substantial amount of charges formed in the bulk of insulation. The only two phases that may show the presence of charge are $0^{\circ}$ and $180^{\circ}$ at which the external voltage is zero. Bearing this and the problems associated with the above methods in mind and also for simplicity, only charges that were trapped reasonably deep were presented. This was done by removing the applied stress after $24 \mathrm{~h}$ of electrical ageing before measuring the volt-off space charge profile. The time span between the removal of voltage and the measurement of the first space charge measurement is approximately $5 \mathrm{~s}$. However, for simplicity, this measurement is referred to here as $0 \mathrm{~h}$ measurement.

The volt-off space charge profile of sample B is shown in figure 8 .

Comparing figure 8 with figures 6 and 7, it can be seen that while smaller in magnitude, the distribution of space charge is generally the same-dominated by negative charge accumulation. This is probably due to fast charge being detrapped during the $5 \mathrm{~s}$ time span needed between the removal of voltage and measurement of space charge. Short circuiting
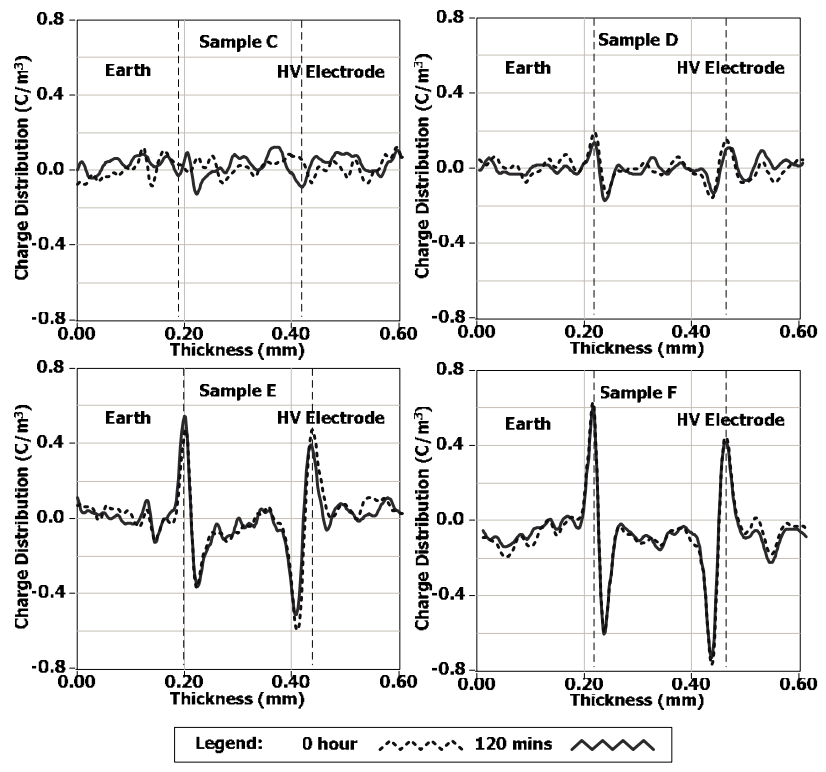

Figure 9. Volt-off charge profiles of samples C to F.

the sample for $2 \mathrm{~h}$ resulted in appreciable decay in the amount of charge but less than what was observed in sample A.

Figure 9 shows the volt-off space charge profiles of the degassed samples after $24 \mathrm{~h}$ ac ageing.

If the frequency of the ac voltage is low, there is more time for charge to be injected, transported and trapped in the bulk before the polarity of the voltage reverses. Therefore, one would expect the amount of charge trapped in a sample to reduce when the frequency increases. Indeed, it was reported that the amount of charge accumulated in LDPE and XLPE reduces as frequency of the applied ac voltage increases $[10,12]$.

However, a quick comparison of the space charge profiles of samples B, C and D suggests otherwise. While at the lowest frequency of $1 \mathrm{~Hz}$ (sample B) one saw the highest amount of charge trapped in the bulk at the end of the scale one did not see the least amount of charge (sample D). In fact, it was sample C, which was aged at $10 \mathrm{~Hz}$ ac voltage, which exhibited negligible charge trapped in the bulk.

One possible explanation is the contribution of space charge on ac ageing is speculated to be mainly due to the fatigue mechanism associated with the electromechanical energy stored or released in trapping/detrapping as well as to the electromechanical forces exerted by the injected charge in each half cycle [20]. Hence, at $50 \mathrm{~Hz}$, sample D was subjected to more cycles of polarity reversal for the given ageing duration compared with both samples B and C. This may result in more rapid degradation which may in turn result in the formation of deeper traps. Another hypothesis is that the deep traps exist prior to electrical ageing. However, our results in [13] do not support this hypothesis. In reality, the effect of frequency on charge accumulation in solid dielectric materials could be more complicated and more research is required.

All the samples tested under ac condition, with the exception of sample $\mathrm{C}$, were dominated by trapped negative charge. This suggests that ac ageing results in the formation of deep traps for negative charge. 
Characteristics of cross-linked polyethylene subjected to ac electric stresses
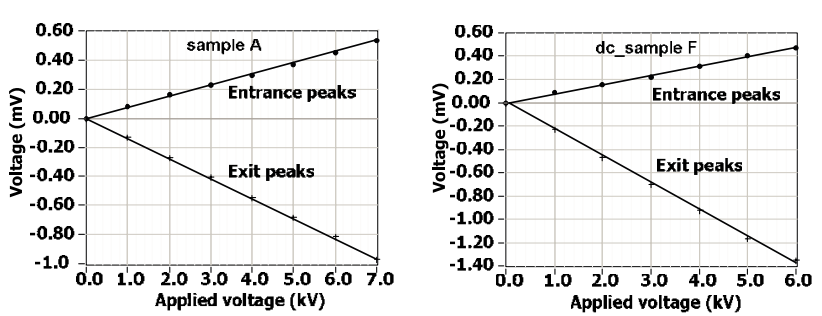

Figure 10. Step voltage test results of sample A and dc-sample F.

It should be noted that in $[10,12]$, space charges were measured while ac voltage was applied on the sample while in the present study the charges measured were those trapped in the sample after the removal of voltage. Therefore, the fast charge may be accountable for the difference in the results.

Comparing the space charge profiles of sample D with samples $\mathrm{E}$ and $\mathrm{F}$ indicates an increase in the amount of accumulated negative charge. This may be attributed to the fact that higher electric stress enhances charge injection and also the process described before.

It was noted that relatively less decay of the trapped charge was observed in samples D, E and F compared with samples A and $\mathrm{B}$. This is probably attributed to the formation of more deep traps as a result of the higher frequency and/or electric stress which supports the hypothesis of deep traps formation.

The second phase of the experiment involved stressing samples B to F under dc electric stress. It was confirmed that the charges trapped in the samples during the first phase of the experiment had decayed to an amount that could no longer be detected by the PEA system.

Before ageing the samples a simple step voltage test was conducted to investigate the threshold stress at which space charge initiates in the samples. Similarly to the procedure adopted earlier, voltage was increased from 0 to 6 or $7 \mathrm{kV}$ at a voltage step of $1 \mathrm{kV}$. Space charge measurements were made at every incremental step. The peak voltages were plotted versus the applied voltage. The results are shown in figure 10 .

Space charge modifies the stresses at the insulation/electrode interfaces which in turn causes the front and rear peaks to deviate from the extrapolated trend line. However, this is not seen in figure 10 suggesting that up to a stress level of $30 \mathrm{kV}_{\mathrm{dc}} \mathrm{mm}^{-1}$, no space charge is formed in the samples. The step voltage test results of dc-samples B to $E$ are similar to that of sample A and dc-sample F and will not be shown in this paper. While the results in this experiment suggest that the threshold voltage is not affected by ac ageing, it is worth pointing out that this deduction only holds true for applied stress of $30 \mathrm{kV}_{\mathrm{dc}} \mathrm{mm}^{-1}$ or less.

The results of the volt-on space charge profiles of dcsamples B to F can be seen in figure 11. Measurements were taken at $0,1,2,4,8$ and $24 \mathrm{~h}$.

With reference to figure 11 , no significant space charge can be seen in dc-samples B to $\mathrm{F}$ at 0 h, i.e. immediately after the step voltage test. This is consistent with the results of the step voltage test.

At $1 \mathrm{~h}$, homocharges can be seen accumulating near the vicinity of both electrodes in all the samples. As in the case of sample A, the formation of these homocharges is believed to be caused by charge injection from the semicon electrodes.

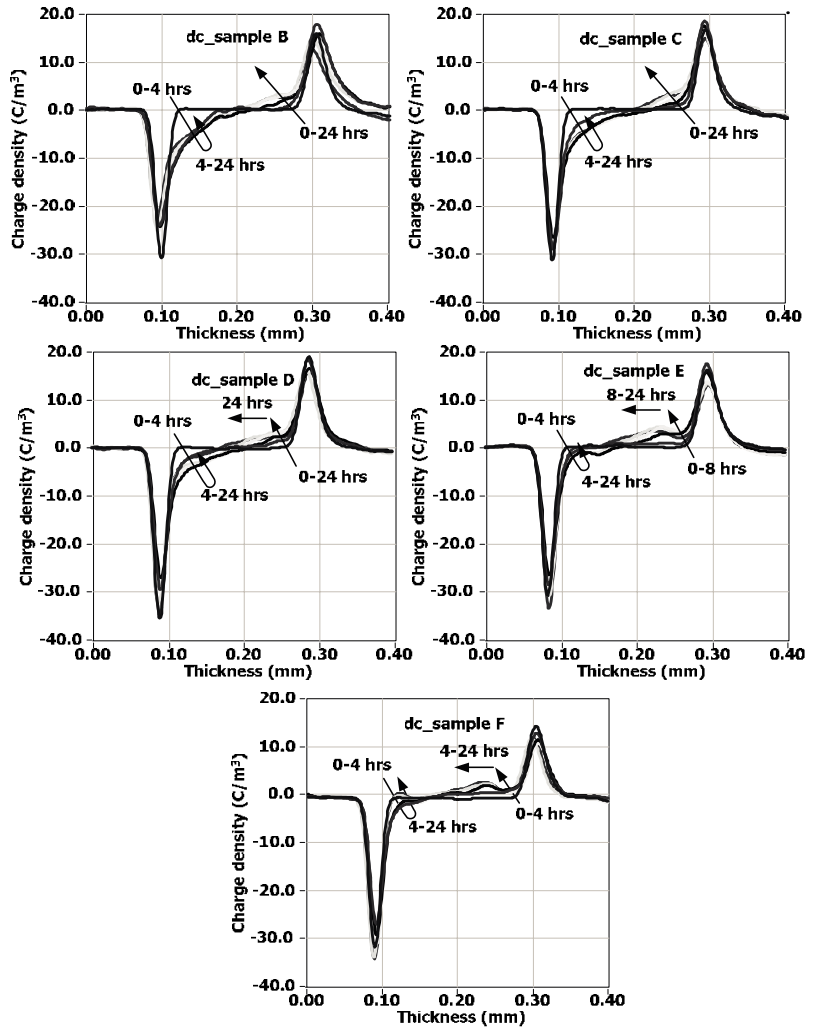

Figure 11. Volt-on charge profiles of dc-samples B to F.

In the case of dc-samples $\mathrm{B}$ and $\mathrm{C}$, an increase in positive homocharge with increasing stress period was observed, with the negative homocharge reaching its maximum amount after $4 \mathrm{~h}$ of ageing.

Similar behaviour can be observed for the negative charge in dc-samples D to F, which appears to reach its maximum amount after $4 \mathrm{~h}$ of ageing. However, in the vicinity of the anode, a positive packet charge can be seen, less obvious in the dc-sample D compare with samples E and F.

It has been reported that packet charge formation may be caused by charge carrier generation under a high electric field (above $100 \mathrm{kV}_{\mathrm{dc}} \mathrm{mm}^{-1}$ ), antioxidant deteriorated by oxidation [21] and local ionization of impurities through salvation by acetophenone [22]. The samples used in this experiment, however, do not contain antioxidant and acetophenone is believed to be largely removed after the degassing process while the stress applied is much lower than $100 \mathrm{kV}_{\mathrm{dc}} \mathrm{mm}^{-1}$. Therefore, formation of packet charge is believed to be caused by the ac ageing during the first phase of the experiment.

Careful comparison of the charge profiles of dc-samples D, $\mathrm{E}$ and $\mathrm{F}$ indicates that the time for packet charge formation is dependent on ac ageing conditions. From figure 11, packet charge only appears in dc-sample D after $24 \mathrm{~h}$ of ageing while it takes only $8 \mathrm{~h}$ in the case of dc-sample $\mathrm{E}$ and $4 \mathrm{~h}$ for dcsample F. It is worth noting that after it initiates, the packet charge tends to move towards the cathode with ageing time as indicated by the arrows. Charge profile in a sample is closely related to charge dynamics (transport, trapping and recombination). The mobility of charge carriers depends not only on trap distribution but also on the local electric field. Charge movement in the sample is complicated, especially 

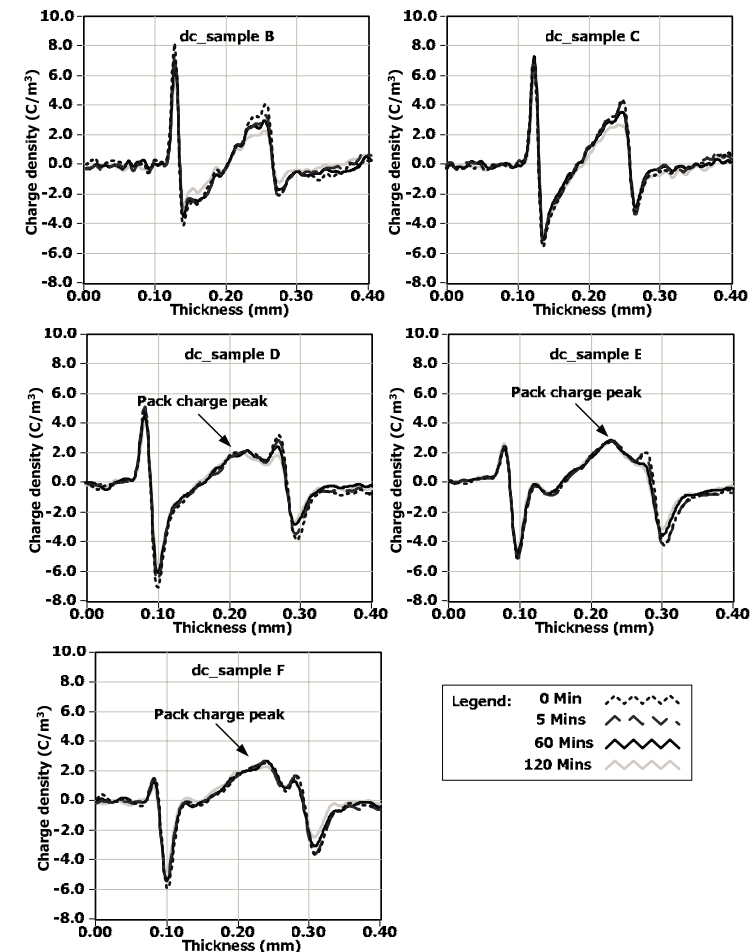

Figure 12. Volt-off charge profiles of dc-samples B to F.

when both positive and negative charges are present. So it is possible that the positive charge packet observed is a result of the above processes.

According to the findings reported in [23], a positive charge packet can be observed only when the positive charge dominates the carriers. A similar conclusion can be drawn by looking at figure 11 , which shows that dc-samples $\mathrm{D}$ to $\mathrm{F}$ are dominated by positive charge. A quick look at figure 12, which shows the space charge profiles of all the samples with the volt-off after the $24 \mathrm{~h}$ of ageing, further confirms this.

The amounts of positive and negative charges trapped in sample A are almost equal as seen in figure $4(b)$. In the case of dc-sample B, positive charge appears to be more dominant, moving more towards the cathode. This effect is even more profound in dc-samples $\mathrm{C}$ and $\mathrm{E}$. This is probably due to the difference in frequencies of the ac stress they were subjected to during the first phase of the experiment.

One way to explain this is that at $1 \mathrm{~Hz}$, dc-sample B was subjected to 10 and 50 times less cycles of polarity reversal when compared with dc-samples $\mathrm{C}$ and $\mathrm{D}$, respectively. Therefore, the effect of ac ageing experienced by dc-sample B can be viewed as comparatively much lesser.

Increasing the ac stress level seems to enhance the effects exhibited in dc-samples B to D. This can be clearly seen in the space charge profiles of dc-samples $\mathrm{E}$ and $\mathrm{F}$ in figures 11 and 12. Another noticeable feature is that as ageing conditions are more severe there are less negative charges adjacent to the cathode as shown in figure 11.

The space charge distributions of dc-samples D to F shown in figure 12 indicate two peaks in the positive charge trapped in the bulk. This is not seen in dc-samples A to C. The second peak, as indicated by the arrow, appears to correspond to the packet charge seen in figure 11 .
The packet charge peak tends to be positioned closer to the cathode when the pre-ageing ac stress is higher. This can be seen by comparing the space charge profiles of dc-samples D to $\mathrm{F}$ in figure 12. Additionally, it seems that negative charges become less with ageing. This may be related to the movement of positive charge. Some of the negative charges may be either neutralized or cancelled out by positive charges from the anode.

Therefore, positive charge propagation may be an indication of material degradation. Indeed, studies have shown some relation between propagation of positive charge and the intrinsic breakdown of insulating materials [24,25]. This may be attributed to the fact that as positive charge propagates closer to the cathode, the interfacial stress at the cathode increases which eventually leads to electrical breakdown.

However, due to limited results no direct relationship can be observed between positive charge propagation and the extent of material degradation or life expectancy. More experimental results are needed before any firm conclusion may be drawn regarding this issue.

The mechanism by which the packet charge forms and moves is believed to be the same as reported in [23]. To summarize, positive charge injected from the anode moves towards the cathode under the influence of the effective stress, $E_{\mathrm{e}}(x)$, given which can be described as

$$
E_{\mathrm{e}}(x)=E_{\mathrm{a}}+E_{\mathrm{c}}(x),
$$

where $E_{\mathrm{a}}=$ the applied stress and $E_{\mathrm{c}}(x)=$ the stress from space charge distribution.

As more positive charge is injected into the bulk, the interfacial stress of the anode reduces and hence the rate of charge injection also reduces. The positive charge then moves towards the cathode under the influence of the effective stress. This probably explains why the positive packet charge in dcsample $\mathrm{F}$ is smaller and penetrates deeper into the bulk.

Reduction of positive charge can be seen in dc-samples A to $\mathrm{C}$ after the voltages were removed for $5 \mathrm{~min}$. On the other hand, the insignificant reduction in positive charge can be observed in dc-samples D to F after voltage removal for the same duration.

In order to confirm this, the integration of the charge densities $(Q)$ across the bulk was calculated using equation (4):

$$
Q(t)=\int_{0}^{d} \rho(x, t) S \mathrm{~d} x,
$$

where $d=$ sample thickness, $x=$ position within the bulk and $S=$ electrode area.

Figure 13 shows the integral of the positive charge of four different samples at various times after the removal of voltage.

It can be seen that the positive charge in sample $A$ and dc-sample $\mathrm{C}$ decays more rapidly than those of dc-samples D and $\mathrm{F}$. This suggests that charges are trapped more deeply in the latter when compared with the former. Hence, it is believed that ac ageing results in the formation of deep traps. This is consistent with the observation in LDPE after ac ageing [13].

It is also noted that the reduction of negative trapped charge is relatively less when compared with that of positive charge. This may imply that ac ageing causes the formation of traps that are more stable for negative charge.

Figure 14, which shows the integral of the negative charge of the same four samples, confirms that negative charge of 


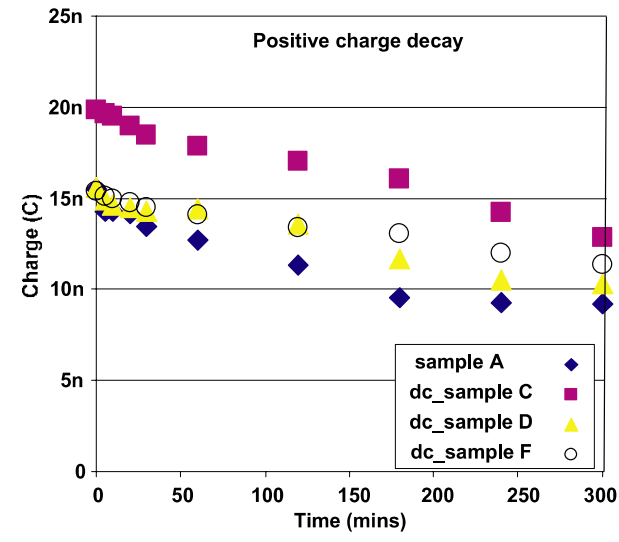

Figure 13. Positive charge decay in selected samples after voltage removal for $5 \mathrm{~h}$.

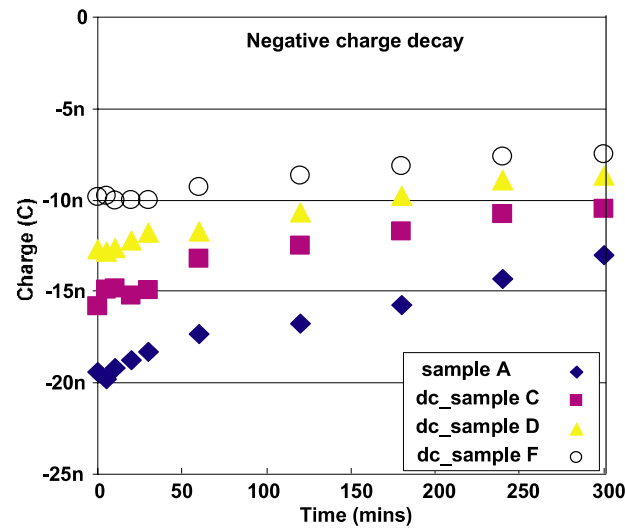

Figure 14. Negative charge decay in selected samples after voltage removal for $5 \mathrm{~h}$.

the dc-samples decays at a slower rate than their positive counterpart.

Charge decay in a sample is governed by several factors such as trap depth and distribution, electric field distribution and temperature. In the present case change in charge decay characteristics after ageing can be attributed to the formation of deep traps after ageing [13].

The concept of fast charge was mentioned earlier. Fast charges, in the context of this study, refer to charges which disappeared almost immediately after the removal of the applied stress. As a time lag of approximately $2-3 \mathrm{~s}$ is needed between the removal of voltage and the measurement of space charge, the ' 0 min' of the volt-off space charge profiles presented thus far does not take fast charge into account. If electrical ageing modifies the trap depth, it may also affect fast charge.

Although it is impossible to measure fast charge in a sample practically, it can however, be computed with equation (5):

$$
\rho_{\text {fast }}(x)=\rho_{\text {accumulated }}(x)-\rho_{\text {volt-off }}(x) \text {. }
$$

Using equations (1) and (2), $\rho_{\text {accumulated }}(x)$ is obtained and subsequently substituted into equation (5). The fast charge profiles are shown in figure 15.

As seen in figure 15, sample A has the highest amount of fast charge among the four samples. This suggests that
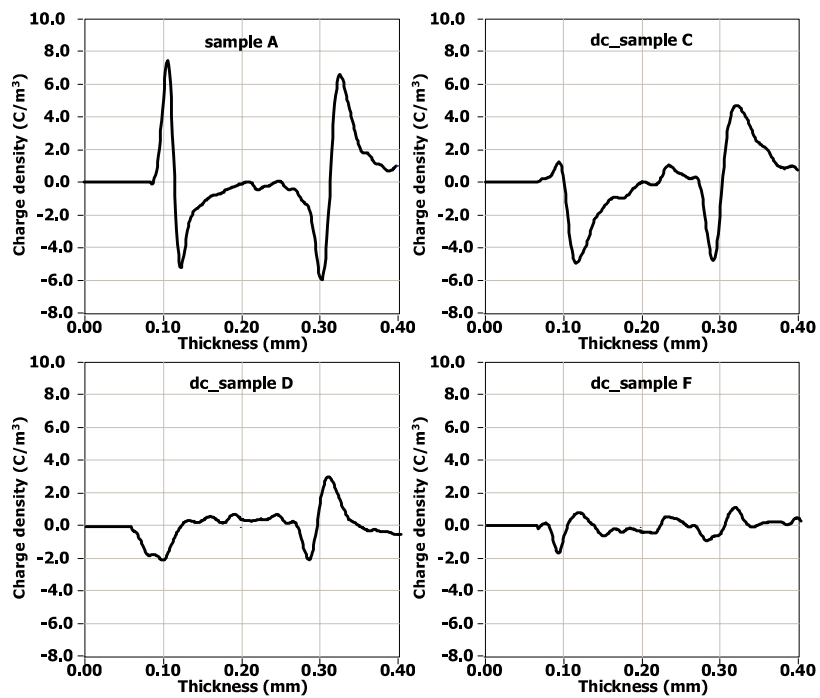

Figure 15. Fast charge selected samples.

sample A has the highest amount of shallow traps. On the other hand, dc-sample $\mathrm{F}$ shows the lowest amount of fast charge which suggests it has the least number of shallow traps. Based on these limited data, it seems that the more severe the electrical ageing, the lesser the amount of fast charge. More experimental work needs to be carried out on different materials before any firm conclusion on fast charge and its relation to material degradation can be drawn.

\section{Conclusion}

Space charge evolution in XLPE planar samples degassed, subjected to dc and ac electric stress has been investigated.

Accumulated space charge, albeit a very small amount, can be seen in most of the samples tested. No significant charge can be observed in the sample subjected to $10 \mathrm{~Hz}$ ac electric stress, while accumulation of negative charges was observed at frequencies of 1 and $50 \mathrm{~Hz}$. It is believed that a combination of charge injection, trapping, formation of deep traps and material degradation caused by ac ageing is accountable for the observed phenomena.

Increasing the applied ac electric stresses increases the amount of charge trapped in the samples, although it is still significantly lower than the amount trapped in the sample subjected to dc electric stress.

The space charge dynamics of the samples used for the investigation of ac space charge were tested again under dc electric stress. The results showed the threshold stress at which space charge initiates was not affected by ac ageing. Furthermore, positive charge becomes more dominant after the samples were aged under ac stress. The higher the frequency and/or magnitude of the ac stress, the more dominant positive charge over negative charge.

As positive charge became more and more dominant, positive packet charge was formed. These were observed in samples that were aged under $50 \mathrm{~Hz}$ ac. The formation and dynamics of the packet charge were explained.

Charge accumulated during the dc stress period was allowed to decay for $5 \mathrm{~h}$. It seems that ac ageing results in 
the formation of deeper traps, particularly for negative charge carriers.

\section{Acknowledgments}

The authors would like to thank the Ministry of Education, Culture, Sports, Science and Technology of Japan for funding the student exchange programme.

\section{References}

[1] Tanaka Y, Chen G, Zhao Y, Davies A E, Vaughan A S and Takada T 2003 Effect of additives on morphology and space charge accumulation in low density polyethylene IEEE Trans. Dielectr. Electr. Insul. $10113-21$

[2] Hirai N, Minami R, Tanaka T, Ohki Y, Okashita M and Maeno T 2003 Chemical group in crosslinking by-products responsible for charge trapping in polyethylene IEEE Trans. Dielectr. Electr. Insul. 10 320-9

[3] Kobayashi K, Ohara T, Ohki Y and Maeno T 1999 Effect of acetophenone on the space charge evolution in LDPE and LLDPE IEEE Conf. Electr. Insul. Dielectric Phenomena (Austin, TX, USA) 27-31

[4] Hirai N, Maeno Y, Tanaka T, Ohki Y, Okashita M and Maeno T 2003 Role of cumyl alcohol and crosslinked structure in homo-charge trapping in crosslinked polyethylene IEEE Conf. Electr. Insul. Dielectric Phenomena (Albuquerque, NM, USA) 213-16

[5] Boudou L, Griseri V, Guastavino J and Dissado L A 2004 Effect of temperature on space charge formation in low density polyethylene-role of antioxidant IEEE Int. Conf. on Solid Dielectric (Toulouse, France) pp 252-5

[6] Doi T, Tanaka T and Takada T 1997 Short interval measurement of space charge distribution in acetophenoen coated low-density polyethylene Int. Conf. on Properties and Applications of Dielectric Materials (Seoul, Korea) pp 810-13

[7] Ho Y F F, Chong Y L and Chen G 2003 Effect of the length of degassing period on the space charge dynamics in XLPE insulation under dc stressing condition Int. Conf. on Insulation Condition Monitoring of Electrical Plant (Chongqing, China) pp 160-3

[8] Ho Y F F, Chen G, Davies A E, Swingler S G, Sutton S J, Hampton R N and Hobdell S 2002 Measurement of space charge in XLPE insulation under $50 \mathrm{~Hz}$ ac electric stresses using LIPP method IEEE Trans. Dielectr. Electr. Insul. 9 362-70

[9] Notingher P, Toureille A, Santana J and Albertini M 1999 Space charge in ac-poled XLPE for HV cables 5th Int. Conf. on Insulated Power Cables (Versailles, France) pp 701-6

[10] Takada T 1999 Acoustic and optical methods for measuring of electric charge distributions in dielectrics IEEE Trans. Dielectr. Electr. Insul. 6 519-47

[11] Montanari G C, Mazzanti G, Boni E and De Robertis G 2000 Investigating ac space charge accumulation in polymers by
PEA measurements IEEE Conf. on Electrical Insulation and Dielectric Phenomena (Victoria, BC, Canada) pp 113-16

[12] Montanari G C, Palmieri F, Mazzanti G, Laurent C and Teyssedre G 2003 AC charge injection ivestigated by means of space charge measurements: theshold and frequency dependence IEEE Int. Conf. on Properties and Applications of Dielectric Materials (Nagoya, Japan) pp 895-9

[13] Chen G, Fu M, Liu X Z and Zhong L S 2005 Ac ageing and space charge characteristics in low density polyethylene polymeric insulation J. Appl. Phys. 97083713

[14] Maeno T, Futami T, Kushibe H, Takada T and Cooke C M 1983 Measurement of spatial charge distribution in thick dielectrics using the pulsed electroacoustic method IEEE Trans. Electr. Insul. 18 433-9

[15] Morshuis P and Jeroense M 1997 Space charge measurements on impregnated paper: A review of the PEA method and a discussion of results IEEE Electr. Insul. Mag. 13 26-35

[16] Maeno T, Hoshino T, Futami T and Takada T 1989 Application of ultrasonic techniques to the measurement of spatial charge and electric field distributions in solid materials Electr. Eng. Japan 86 58-64

[17] Fu M, Chen G, Davies A and Head J 2001 Space charge measurements in cables using the PEA methodsignal data processing consideration IEEE Int. Conf. on Solid Dielectrics (Eindhoven, Netherlands) pp 219-22

[18] Liu R, Takada T and Takasu N 1993 Pulsed electroacoustic method for measurement od space charge distribution in power cables under both dc and ac electric fields J. Phys. D: Appl. Phys. 26 986-93

[19] Wang X, Yoshimura N, Tanaka Y, Murata K and Takada T 1998 Space charge characteristics in cross-linking polyethylene under electrical stress from dc to power frequency J. Phys. D: Appl. Phys. 31 2057-64

[20] Mazzanti G, Montanari G C and Dissado L A 1999 A space-charge life model for the ac electrical ageing of polymers IEEE Trans. Dielectr. Electr. Insul. 6 864-75

[21] Kon H, Suzuoki Y, Mizutani T, Ieda M and Yoshifuji N 1996 Packet-like space charges and conduction current in polyethylene cable insulation IEEE Trans. Dielectr. Electr. Insul. 3 380-5

[22] Hozumi N, Takeda T, Suzuki H and Okamoto T 1998 Space charge behavior in XLPE cable insulation under 0.2-1.2 $\mathrm{MV} / \mathrm{cm}$ dc fields IEEE Trans. Dielectr. Electr. Insul. 5 82-90

[23] Chen G, Tay T Y G, Davies A E, Tanaka Y and Takada T 2001 Electrodes and charge injection in low-density polyethylene IEEE Trans. Dielectr. Electr. Insul. 8 867-73

[24] Fukuma M, Wadamori M, Nagao M, Kosaki M, Fukunaga K and Maeno T 2001 Short interval space charge and external circuit current measurements in LDPE film in high temperature region IEEE Int. Symp. on Electrical Insulating Materials (Himeji, Japan) pp 497-500

[25] Matsui K, Tanaka Y, Takada T and Maeno T 2004 Space charge observation in various types of polyethylene under ultra-high dc electric field IEEE Int. Conf. on Solid Dielectrics (Toulouse, France) vol 1, pp 201-4 